\title{
An Adaptive Growing Hierarchical Self Organizing Map for Network Intrusion Detection
}

\author{
Dennis Ippoliti ${ }^{1}$ and Xiaobo Zhou ${ }^{1,2}$ \\ Department of Computer Science, University of Colorado at Colorado Springs, USA ${ }^{1}$ \\ PhD Program, College of Computing and Information Sciences, Rochester Institute of Technology, USA ${ }^{2}$ \\ Corresponding email: zbo@cs.uccs.edu
}

\begin{abstract}
The growing hierarchical self organizing map (GHSOM) has been shown to be an effective technique to facilitate anomaly detection. However, existing approaches based on GHSOM are not able to adapt online to the ever-changing problem domain of network intrusion. This results in low accuracy in identifying network intrusions, particularly "unknown" attacks. In this paper, we propose an adaptive GHSOM based approach (A-GHSOM) to network intrusion detection. It consists of four significant enhancements: enhanced threshold-based training, dynamic input normalization, feedback-based quantization error threshold adaptation, and prediction confidence filtering and forwarding. We test the capability of the A-GHSOM approach for intrusion detection using the KDD'99 dataset. Extensive experimental results demonstrate that compared with eight representative intrusion detection approaches, A-GHSOM achieves significant overall accuracy improvement and significant improvement in identifying "unknown" attacks while maintaining low false-positive rates. It achieves an overall accuracy rate of $\mathbf{9 9 . 6 3 \%}$, and $\mathbf{9 4 . 0 4 \%}$ accuracy rate in identifying "unknown" attacks while the false positive rate is $\mathbf{1 . 8 \%}$.
\end{abstract}

\section{INTRODUCTION}

Machine learning approaches have been used for anomaly detection [1], [4], [10]. In particular, approaches based on self-organizing maps (SOMs) of artificial neural networks have shown effectiveness at identifying "unknown" attacks [8], [9], [12]. Those studies focused on the training process of SOMs [8], the exploration of training data composition [9], [12], and proper feature selection for training sets and the effect of map topology [9]. However, the effectiveness of using traditional SOM models is limited by the static nature of the model architecture. The size and dimensionality of the SOM model is fixed prior to the training process and is determined by trial and error [9], [12]. There is no systematic method for identifying an optimal configuration. One approach that attempts to overcome this issue is the growing hierarchical self organizing map (GHSOM) [3], [11].

GHSOM is an SOM model that does not use a predetermined map topology. Instead, the size and the dimensionality of the map dynamically grow during the training process to optimally fit the training set based on user defined parameters. The main advantage of a GHSOM over a traditional SOM is that trial and error are eliminated from the training process. An ideal topology is formed unsupervised based on the training data. Additionally, hierarchal relationships in the training data are discovered and modeled in the final configuration. However, although GHSOM has shown some promise in network intrusion detection [11], it does not account for concept drift. That is, although the topology is modeled to fit the training set, it is not adapted online to account for changes to live data that occur over time. There is no ability to adapt the model as live data is processed. Over time, subtle changes in legitimate traffic patterns as well as vulnerability to previously "unknown attacks" require updates to maintain accuracy.

In this paper, we propose an adaptive growing hierarchical self organizing map (A-GHSOM) that adapts online to changes in the input data over time. We do this with four significant enhancements. First, we develop a threshold based training process. The training process expands the GHSOM model to fit the training set. Instead of using the mean quantization error as a control parameter, we establish a new parameter of threshold error value that is more suitable to the network intrusion detection problem.

Second, we use a dynamic input normalization process that monitors the range of observed values of input connections and uses the information to adapt the map scale during normalization online.

Third, we apply feedback-based quantization error threshold adaptation. Quantization error is a measure of how closely an evaluated connection fits its matched neuron in the map. We use an error threshold that adapts over time to identify new attacks that may be initially matched to "normal" nodes in a GHSOM but are indeed malicious.

Finally, we design a prediction confidence filtering and forwarding mechanism to identify traffic patterns that are beyond the ability of a content oblivious system to evaluate. These connections can be filtered or forwarded to a contentaware intrusion detection system for further evaluation.

We conduct experiments using the KDD'99 dataset [7]. Many people argue to use live data for network intrusion detection as the KDD'99 dataset is not completely effective. Recent studies in [6], [10], [9], [11], [17] use the KDD'99 dataset for network intrusion detection since it is a major publicly available dataset. We use the KDD'99 dataset for performance comparison of A-GHSOM with related approaches.

Our baseline A-GHSOM approach achieves $92.21 \%$ accuracy and $0.58 \%$ false positive. Applying those four significant enhancements, the accuracy of the integrated A-GHSOM approach increases to $99.63 \%$ while false positive rate is just slightly increased to $1.8 \%$. It is able to identify a subset of connections that are virtually indistinguishable based on the 
data available in the KDD'99 dataset. By identifying those connections, it increases the accuracy on detecting previously "unknown" attacks to $94.04 \%$ while maintaining $1.8 \%$ false positive rate. When examining individual attack categories of Denial of Service (DoS), Probe, Remote to Local (R2L), and User to Root (U2R) it achieves accuracy of $99.82 \%, 99.58 \%$, $92.66 \%$ and $87.14 \%$ respectively. Compared to eight representative intrusion detection approaches, A-GHSOM significantly increases the accuracy with low false positive rates.

\section{RELATED WORK}

Network intrusion detection has been an active area of research and development. Many approaches have used machine learning techniques to address the issue of anomaly detection. For instance, K-nearest neighbor techniques were used to compute the approximate distance between different data points with vectors being classified by a majority vote of its neighbors [1], [4], [10]. Naive Bayes networks were used to calculate statistical probabilities that certain features can indicate traffic classification [13], [14]. Decision tree methods were used to classify a sample through a sequence of decisions used to influence future decisions [1], [2]. Support vector machine approaches were often used for examining the results of intrusion detection approaches [4], [9], [15], [16].

Neural Network approaches use computational models that try to simulate functional aspects of biological neural networks. Approaches based on SOMs have shown effectiveness at identifying both known and "unknown" attacks [8], [9], [12]. Authors in [12] used a hierarchy network built on an SOM architecture with no neighborhood or transfer functions. Experiments were conducted with a variety of random training sets. Five training sets were used, each one consisting of a different distribution of connection classes. Each layer of the hierarchy was trained on individual and exclusive feature sets.

The work in [9] examined several approaches related to the application of SOMs to intrusion detection. Experiments were conducted with three different partitions of the training data, the entire 10 percent set, normal only connections, and a filtered set consisting of equal numbers of attack vs. normal connections. It also compared the effectiveness of using only 6 basic features to using all 41 features available in the sample data. Networks of differing complexity were tested.

In those traditional SOM based approaches, the size and dimensionality of the model is fixed prior to the training process. In [11], a GHSOM was used to overcome the limitation. The GHSOM is a model with a hierarchical structure composed of independent growing SOMs. The initial map size is very small. The map grows until the mean quantization error is below a predetermined training parameter. The map is only adapted during training. Once training is complete and live data is applied, no adaptation or new learning occurs.

Our approach differs from the existing approaches in important ways. We design a threshold based training process. We add online adaptation for input normalization. We use operator feedback to further adapt the model online and to identify connection patterns that deserve content-aware examination.

\section{Growing Hierarchical Self Organizing MaP}

GHSOMs are used against high dimensional data in neural networks [3] and in network intrusion detection [11]. In a GHSOM, the size and the dimensionality of the map architecture are determined during the training phase. Figure 1 depicts a GHSOM architecture after off-line training. The initial map size is very small, usually a single layer $2 \times 2$. During the training process, the map grows both vertically and horizontally until the training process is complete. Two configurable parameters are used, $\delta_{E}$ and $\delta_{D} . \delta_{E}$ represents the target quantization error for the map, and $\delta_{D}$ represents the maximum dimensionality of a single layer. After each training iteration, the deviation of the input data (quantization error) is computed. The map grows horizontally by adding rows and columns to the map to reduce quantization error. It grows vertically by adding child layers to parent layers that exceed the maximum dimensionality specified by $\delta_{D}$. The process continues until the quantization error of the map is less than $\delta_{E}$. It is important to note that at the end of the training process, each layer and sub-layer can have a different number of maps and sub-maps with varying dimensionality.

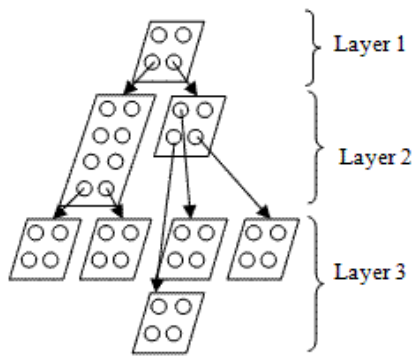

Fig. 1. A GHSOM architecture after off-line training.

\section{Threshold-Based Training Process}

The training process is executed in two cycles, a learning cycle and a growth cycle. During the learning cycle, the GHSOM nodes represent the various input patterns. During the growth cycle, the GHSOM architecture grows both horizontally and vertically. Two cycles continuously alternate until the training process is complete.

During the learning cycle, each input pattern $j$ is represented by an $n$-dimensional input vector $V_{j}$,

$$
V_{j}=\left[v_{j}^{1}, v_{j}^{2}, \ldots, v_{j}^{n}\right] .
$$

The initial map size is set to a single layer of $2 \times 2$. Each node $j$ is assigned an $n$-dimensional weight vector $W_{j}$, which is initialized with random values as follows:

$$
W_{j}=\left[w_{j}^{1}, w_{j}^{2}, \ldots, w_{j}^{n}\right] .
$$

The weight vectors of the best matching unit and its neighborhood are adjusted such that for each node $j$ in the neighborhood, $W_{j}$ is adjusted according to

$$
W_{j}=W_{j}+\alpha\left(W_{j}-V_{j}\right)
$$


where $\alpha$ represents the learning rate. The initial size of the neighborhood is large. This adjustment step is repeated with an increasingly smaller neighborhood size until the neighborhood only contains the best matching unit.

After each learning cycle, there is a growing cycle. The topology of the GHSOM is adapted during the growing cycle. The quantization error is analyzed for the GHSOM after each training cycle. For each input pattern $j$, the quantization error vector $Q E_{j}$ is presented as follows:

$$
Q E_{j}=\left[q e_{j}^{1}, q e_{j}^{2}, \ldots, q e_{j}^{n}\right]
$$

where $q e_{j}^{k}=\left|w_{j}^{k}-v_{j}^{k}\right|, k=1,2, \ldots n$.

The threshold error value is calculated using the quantization error vector as follows:

$$
\begin{aligned}
& T E V_{j}=\sum_{k=0}^{n} f(k) \\
& f(k)= \begin{cases}q e_{j}^{k} & \text { if } k>\tau_{1} \\
0 & \text { if } k \leq \tau_{1}\end{cases}
\end{aligned}
$$

Here, $\tau_{1}$ is a threshold error value that controls how closely an element of an input vector must match an element of a weight vector before it is no longer factored into the threshold error value. The node with the highest threshold error value is considered the highest error node.

The rationale behind Eq. (6) is that the input pattern of an anomalous connection often matches the input pattern of a normal connection very closely except for one or two parameters. Existing GHSOM models use parameters such as the mean quantization error of the vector, or Euclidean distance between vectors [3], [11]. However, they would not be as effective as using the threshold error value given by Eq. (6). For example, consider three vectors representing two connections and one weight vector. Connection- 1 and Connection-2 are each compared to the weight vector. Figure 2 shows the individual quantization errors for each data point in the vectors. If we were to use mean quantization error or Euclidian distance to evaluate Connection-1 and Connection-2, we would find that the mean quantization error and Euclidian distance are higher for Connection-1. However, it is obvious that Connection-2 has an anomalous value in the vector. Instead, by using the threshold error value, we are able to capture this anomaly condition. For effective anomaly detection, we are interested in not only the aggregate small discrepancies, but also identifying large single discrepancies.

Once the highest error node is identified, its threshold error value is compared to the growing threshold $\delta_{E}$. If it is greater than $\delta_{E}$, the map is expanded. During expansion, the number of nodes in the sub-map containing the highest error node is compared to the growing threshold $\delta_{D}$. If it is greater than $\delta_{D}$, a new sub-map is created subordinate to the highest error node. If less, a new column or row of neurons is inserted between the highest error node and its most dissimilar neighbor. Their weight vectors are initialized as the mean of their neighbors' weight vectors.

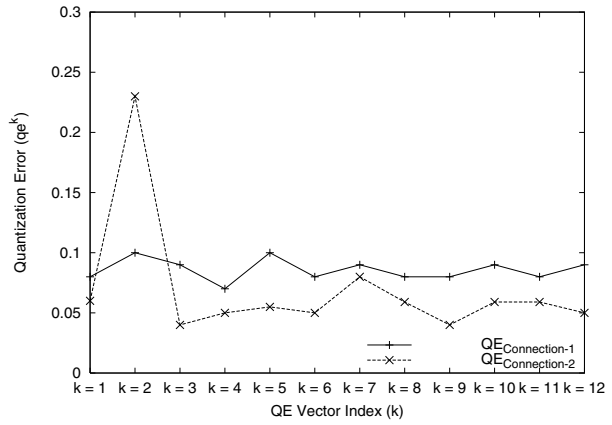

Fig. 2. Quantization error vectors of two sample connections.

\section{DYNAMIC INPUT NORMALIZATION}

\section{A. Data normalization and the effect of scale}

In a GHSOM, data is normalized to account for differences in scale between two different data points. As an example consider that the two data points are height and weight, height being 5 6 feet and weight being 100 200 pounds. Weight would always take precedence over height if the data is not normalized. We propose to account for changes in the data scale over time. By dynamically updating the scale of the input data, we are able to highlight the true distance between different values of the same feature. This is particularly useful when identifying "anomalous" behavior.

To capture relative distance between two different values of the same feature, we adjust the input pattern so that all values are in the range of 0 to 1 using simple linear scaling for the normalization. We consider the "distance" to be how different these two values are from one another. The smaller the distance, the more similar the values are considered.

\begin{tabular}{|c|c|c|c|c|}
\hline Minimum & Maximum & Value-1 & Value-2 & Normalized Distance \\
\hline \hline 1 & 16 & 8 & 14 & 0.4 \\
1 & 32 & 8 & 14 & 0.194 \\
1 & 64 & 8 & 14 & 0.095 \\
1 & 128 & 8 & 14 & 0.047 \\
1 & 256 & 8 & 14 & 0.023 \\
\hline
\end{tabular}

TABLE I

EFFECT OF SCALE ON NORMALIZED DISTANCE.

In Table I, the reference range used to normalize input values varies from $1 \sim 16$ to $1 \sim 256$. For each reference range, two values 8 and 14 are normalized based on the range and their normalized distance is calculated. When the reference range is $1 \sim 16$, two values are considered very different from each other as the normalized distance is 0.4 . When the reference range is $1 \sim 256$, they are considered very similar as the normalized distance is 0.023 .

In an intrusion detection problem domain, there are several data points that the scale will be known ahead of time. Some points are in the scale of $0 \sim 1$, while some others are in $0 \sim 255$. However, in the KDD'99 dataset, 17 of the 42 data points have an undefined scale. Furthermore, the scale in the training data is not the same as the scale in the live data. Therefore, 
scaling done during the training process will not be accurate when applied to the live data. In our A-GHSOM approach, we propose an adaptive input normalization approach that automatically tunes the scaling parameters and weight vectors online based on the observed minimum and maximum values.

\section{B. Dynamic input normalization process}

The KDD'99 data contains both numeric and symbolic values. In the dynamic input normalization process, numeric inputs are normalized to values between 0 and 1 . Flag values are normalized to integer values greater than 1 so that the possible outcomes of comparing two flag values are only differences equal to zero or greater than or equal to one. All other values are normalized according to the normalization function. When compared to the weight vector, differences close to 0 are considered extremely similar with 0 being identical, differences close to 1 are considered very different (differences $\geq 1$ are considered infinitely different).

The normalization function $F(x)$ is given as follows:

$$
F(x)= \begin{cases}\frac{x-\min (t)}{\max (t)-\min (t)} & \max (t) \neq \min (t) \\ 0 & \max (t)=\min (t)\end{cases}
$$

where $x$ is a data point in the input pattern, $\min (t)$ and $\max (t)$ are the expected minimum and maximum values for that data point at time $t$ respectively. Prior to training, the minimum and maximum values in the training set are calculated for each data point. These values are used to normalize the input patterns at time $t$. As the effect scale can have significant impact on identifying the variance, the ideal situation is to have the most narrow range possible to normalize the data and therefore amplify the true difference between vectors. Over time, if no input data is as low as the minimum, raise the minimum. If no data input is as high as the maximum, lower the maximum. As live traffic is processed, at periodic intervals from time $t$ to time $t+n$ where $n$ is the number of records processed, the observed minimum and maximum values are recorded as $o b s_{\min }(t)$ and $o b s_{\max }(t)$ respectively. At time $t$, the minimum and maximum values are adjusted according to the following equations.

$$
\begin{aligned}
& \max (t+n)=\min (t)+R(t) \cdot e^{-\alpha}+\beta \max _{\Delta}(t) . \\
& \min (t+n)=\max (t)-R(t) \cdot e^{-\alpha}-\beta \min _{\Delta}(t) .
\end{aligned}
$$

In equations 8 and 9 , we have

$$
\begin{aligned}
\max _{\Delta}(t) & = \begin{cases}o b s_{\max }(t)-\max (t) & o b s_{\max }(t)>\max (t) \\
0 & o b s_{\max }(t) \leq \max (t)\end{cases} \\
\min _{\Delta}(t) & = \begin{cases}\min (t)-o b s_{\min }(t) & o b s_{\min }(t) \leq \min (t) \\
0 & o b s_{\min }(t)>\min (t)\end{cases} \\
R(t) & =\max (t)-\min (t) .
\end{aligned}
$$

Control parameters $\alpha$ and $\beta$ have values between 0 and 1. $\alpha$ controls the exponential growth/decay rate. $\beta$ controls the differences between the current used $\min / \max$ and the observed $\mathrm{min} / \mathrm{max}$ is used. Figure 3 demonstrates the relationship between the observed $\min / \max$ values and the adapted $\mathrm{min} / \mathrm{max}$ values. They represent a subset of the "destinationbytes" feature of the KDD dataset. The x-axis represents the time interval that the measurement was recorded. The $y$-axis

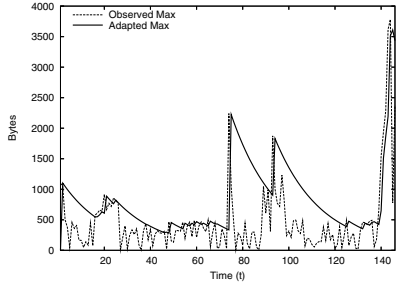

(a) Maximum values

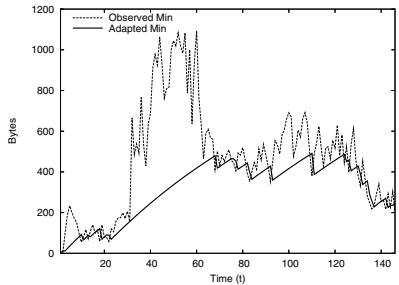

(b) Minimum values.
Fig. 3. Examples of adapted maximum and minimum values.

represents the min or max value in bytes. The plotted series represent the relationship between the observed values and the adapted values with $\alpha=0.05$ and $\beta=1.0$. The adapted values are used for scaling during the normalization process.

\section{Vi. Feedback-based Threshold Adaptation}

A-GHSOM is further enhanced by use of feedback-based quantization error threshold adaptation. It adaptively adjusts thresholds for each node as input patterns are applied and add new nodes when appropriate. Each node is assigned two initial threshold parameters. $\tau_{1}$ is used to calculate the threshold error value. $\tau_{2}$ is used as an upper limit on the acceptable total quantization error and used to calculate the quantization error boundary (QEB) for a selected node as follows.

$$
Q E B_{j}= \begin{cases}0 & t q e_{j}<\tau_{2} \\ 1 & t_{q e_{j}}>=\tau_{2}\end{cases}
$$

where $t q e_{j}=\sum_{k=1}^{n} q e_{j}^{k}$

During live intrusion detection, input patterns are applied to the A-GHSOM and the best matching unit is selected. However, because of the size of the problem space, there is no guarantee that the best matching unit is a good match to the input pattern. The only guarantee is that it is a better match than all the other nodes in the neural network. The threshold error value and quantization error boundary are used to determine if the connection being processed is within thresholds. If and only if they are identically equal to 0 , is the pattern considered within thresholds.

Each node in the final A-GHSOM is marked either "normal", "unmarked", or "attack". As patterns are examined, they are mapped to one of the three node types and considered within thresholds or not. Results are then used to make predictions to identify the suspected connection type, either "normal" or "attack". Any pattern that is not within thresholds is identified as a suspected attack. Even though its best matching unit is labeled "normal", the fact that its threshold error value or its quantization error boundary is greater than 0 means this connection is anomalous in nature and it is assumed to be an attack. Likewise, any patterns mapped to a best matching unit of type "unmarked" are patterns that have not been previously identified and are suspected attack. The actual connection type is then compared to the suspected connection type and a result of "correct" or "incorrect" is identified.

\section{CONFIDENCE FILTERING AND FORWARDING}

The fourth enhancement of the A-GHSOM approach is a prediction confidence filtering and forwarding mechanism. It 
monitors the neuron consistency and accuracy and uses those measures to develop a neuron confidence rating. It then uses the rating to identify an appropriate action for the system to take regarding predictions made by nodes with which the system has low confidence.

In a traditional GHSOM, individual neurons and regions of the map are organized to classify connections. All connections matched to identical regions of the map will be classified as the same. However, in A-GHSOM, due to the addition of dynamic input normalization and feedback based threshold adaptation, this is not the case. It is possible for two connections to be matched in an identical region on the map, but be placed into different classification categories depending on their threshold error value and quantization error boundary value. Neurons that are essentially trained to agitate on normal connections can indicate attack and vice versa. We monitor the frequency that each neuron predicts each connection class and use this condition to calculate a consistency rating. After each connection is processed at time $t$ and a prediction made by neuron $j$, consistency for the predicting node is calculated according to Eq. (13).

$$
\begin{aligned}
& \operatorname{Attack}_{t}^{j}=\frac{\lambda\left(\text { Attack }_{t-1}^{j}+\right.\text { PREDICT }}{\lambda+1} . \\
& \text { Normal } \left._{t}^{j}=\frac{\lambda\left(\text { Attack }_{t-1}^{j}+\right.\text { PREDICT }}{\text { Normal }}\right) . \\
& \text { Consistancy }_{t}^{j}=\frac{\max \left(\text { Attack }_{t}, \text { Normal }_{t}\right)}{\text { Attack }_{t}+\text { Normal }_{t}} .
\end{aligned}
$$

where $\lambda$ is the size of the history, $P R E D I C T_{\text {Attack }}=1$ if an attack is predicted and 0 otherwise, and $P R E D I C T_{\text {Normal }}=$ 1 - PREDICT $T_{\text {Attack }}$.

Because A-GHSOM also expects feedback from a network operator, we are able to estimate an accuracy rating for each node. As the amount of feedback received by the system is limited, the system assumes that its predictions are correct in the absence of feedback. PREDICT $T_{\text {Accurate }}$ is equal to 0 if corrective feedback is received and 1 otherwise. Accuracy for predicting node $j$ is calculated according to Eq. (14).

$$
A c c_{t}^{j}=\frac{\lambda\left(A c c_{t-1}^{j}\right)+P R E D I C T_{\text {Accurate }}}{\lambda+1} .
$$

We use a combination of consistency and accuracy to calculate a confidence rating as follows.

$$
\text { Confidence }_{t}^{j}=\frac{\lambda\left(\text { Confidence }_{t-1}^{j}\right)+\text { Acc }_{t}^{j}+\text { Consistancy }_{t}^{j}}{\lambda+1} .
$$

We identify nodes that have low confidence and exclude traffic that they are processing.

\section{EXPERIMENTAL RESULTS AND ANALYSIS}

The KDD'99 dataset contains records describing TCPconnections. The dataset includes normal connections as well as 23 different types of attacks belonging to four categories: Denial of Service (DOS), Probe attacks, User to Root (U2R), and Remote to Local (R2L). The dataset includes a training set, and two test sets: the "whole" set and the "corrected" set. The "corrected" set includes 17 attack types that are not sampled in the training set and thus are "unknown" to a trained model. For all experiments, we trained our A-GHSOM model on the training set and tested it against the "corrected" set.

\begin{tabular}{|c|c|}
\hline CATEGORY & ACCURACY \\
\hline \hline Overall & 92.21 \\
Known & 92.81 \\
Unknown & 10.95 \\
DOS & 98.48 \\
R2L & 7.66 \\
U2R & 51.43 \\
Probe & 78.83 \\
\hline
\end{tabular}

TABLE II

ACCURACY OF THE BASELINE A-GHSOM.

\begin{tabular}{|c|c|}
\hline CATEGORY & ACCURACY \\
\hline \hline Overall & 93.53 \\
Known & 99.37 \\
Unknown & 21.27 \\
DOS & 97.59 \\
R2L & 35.11 \\
U2R & 84.29 \\
Probe & 99.06 \\
\hline
\end{tabular}

TABLE III

ACCURACY OF A-GHSOM WITH THRESHOLD BASED TRAINING.

For each connection, a decision is made to process the connection or to forward to the operator based on system confidence. For each connection processed, a classification of "attack" or "normal" is made. The performance metrics are the accuracy rate and the false positive rate. The accuracy rate is the ratio of the total number of correct attach predictions over the total number of attacks processed. The false positive rate is the ratio of the total number of false positive predictions over the total number of normal connections processed.

To establish a baseline for performance comparison, we processed the corrected dataset without applying any of the four enhancements. In this configuration, we train the AGHSOM map using $\delta_{E}$ and $\delta_{D}$ of 0.5 and 100 respectively. Without using thresholds and adaptations, connections are simply classified based on the node they are mapped to. Table II gives the overall accuracy rate, individual rates for each attack category, and unknown and known attacks. The overall false positive rate is $0.58 \%$. Next, step by step we study the impact of four enhancements of the A-GHSOM approach on the performance of intrusion detection.

\section{A. Impact of the threshold-based training}

In this experiment, the threshold-based training process is applied, but without adaptations. Furthermore, dynamic input normalization is not used and no operator feedback is assumed. Initial default threshold values for $\tau_{1}$ and $\tau_{2}$ are set to 0.5 and 1.0 respectively, but not adapted dynamically.

Table III shows the overall accuracy rate, individual rates for each attack category, and unknown and known attacks. We observed that without any adaptation, the overall accuracy is improved slightly while significant improvements are achieved in the categories of R2L, U2R, PROBE, and Unknown. The improvements come at a cost to the false positive rate that increases to $1.73 \%$. 

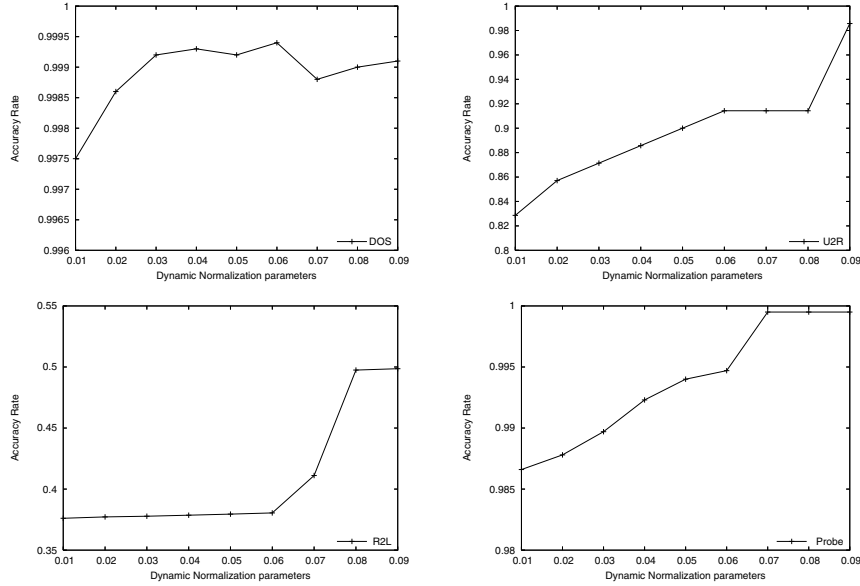

(a) On DOS accuracy.

(c) On R2L accuracy. (b) On U2R accuracy.

(d) On PROBE accuracy.

Fig. 4. Impact of the dynamic input normalization.

\section{B. Impact of the dynamic input normalization}

We study the impact of the dynamic input normalization on the performance of A-GHSOM approach. We dynamically change the normalization parameters $\alpha$ and $\beta$ from .01 to .09 for every 15000 connections. The parameters are used to control the level of aggressiveness that the dynamic normalization scale is adapted. The more aggressive the adaptation, the larger the absolute variance between two vectors will be. With the absolute variance between vectors increased, connections are more likely to produce quantization error profiles that exceed thresholds. The overall objective in applying this technique is to amplify subtle differences between connections, particularly "unknown" connections, and then use this amplification to increase overall intrusion detection accuracy.

Figure 4 shows that the system with the enhancement overall improves the accuracy significantly for all four attack categories. However, the A-GHSOM model is too sensitive to the normalization parameters. Increased accuracy comes at a cost of higher false positive rate. Figure 5 depicts the relative operating characteristics (ROC) diagrams that show the relationship between the accuracy and the false positive rate of four attack categories. It shows the false positive rate can be out of control. This is due to the fact that the dynamic input normalization amplifies the variance between vectors. Small differences can register high quantization errors. The threshold-based training process interprets those high errors as anomalous traffic. While many more attacks are identified correctly, more normal connections are flagged as attacks.

\section{Impact of the feedback-based threshold adaptation}

Until now, no expert feedback is used. Next, operator feedback is used to further enhance the accuracy of intrusion detection. We use a control parameter of feedback probability to determine the likelihood that the system will be notified that it has made an error. We acknowledge that under normal operating conditions, it is unlikely that an operator will be able

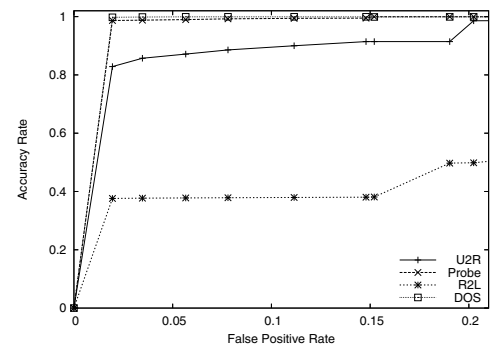

Fig. 5. ROC diagrams of A-GHSOM with the dynamic input normalization.

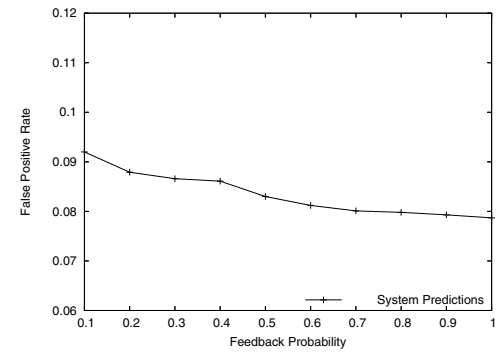

Fig. 6. False positive rate.

to respond to every alarm or to identify every missed attack in a timely manner. In the experiments, the feedback probability is equal to the percentage of mistakes that the system makes and the operator is able to provide feedback on. In the absence of feedback, the system assumes its prediction was correct.

With the adaptive thresholds, we are able to capitalize on the dynamic input normalization and bring false positives down to more reasonable levels. When the system receives feedback that it has made an incorrect prediction, the thresholds are adjusted so that future similar vectors are more likely to be handled correctly. This adjustment is made on a per node basis so that only nodes making inaccurate predictions are adapted. In the experiment, the feedback probability varies from $1 \%$ to $10 \%$. Dynamic input normalization parameters $\alpha$ and $\beta$ are set to 0.04 per every 15000 connections.

Figure 6 shows the false positive rate with the feedbackbased threshold adaptation. Compared to the system that does not use the feedback-based threshold adaptation, the false positive rate is much lower. However, it varies around $8 \%$ to $10 \%$, which is still higher than desired.

\section{Impact of the integrated A-GHSOM approach}

Figure 7 demonstrates the effect of the integrated AGHSOM approach on intrusion detection. The system is adapted every 20000 connections using different parameters. Input normalization parameters $\alpha$ and $\beta$ are set to .03 , and the confidence forwarding probability is $80 \%$. In the experiment, $91.9 \%$ of the connections are classified by the integrated approach. $8.1 \%$ of the connections are selected for forwarding.

The integrated approach achieves an overall accuracy rate of $99.63 \%$ while maintaining the false positive rate of $1.8 \%$. All four categories of attacks are predicted with above $87 \%$ accuracy. We see the sharpest increase in the accuracy of 


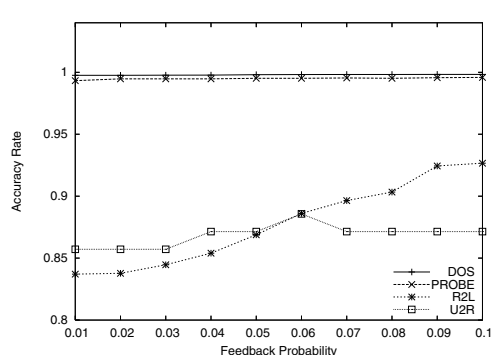

(a) Accuracy by categories.

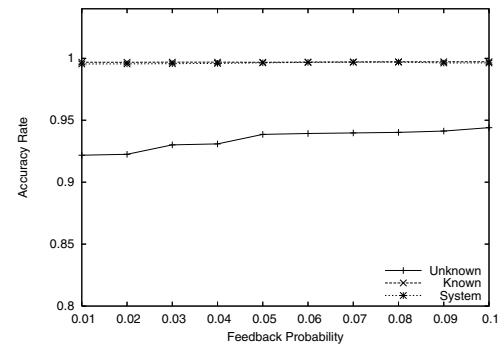

(b) Accuracy by known \& unknown.

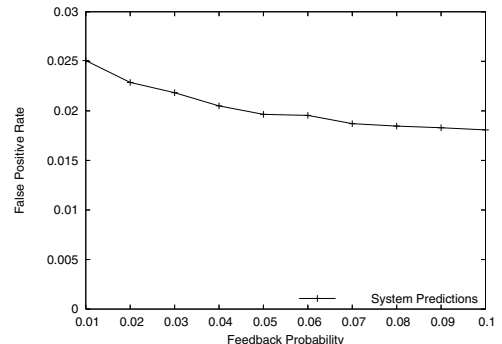

(c) False positive rate.

Fig. 7. Impact of the integrated A-GHSOM approach on detection accuracy and false positive rate.

\begin{tabular}{|c|c|c|}
\hline Approach & Accuracy & False Positive \\
\hline \hline A-GHSOM & $99.63 \%$ & $1.8 \%$ \\
GHSOM [11], year 2008 & $90.87 \%$ & $2.69 \%$ \\
Bouzida [1], year 2004 & $91.89 \%$ & $0.48 \%$ \\
Sarasamma [12], year 2005 & $93.46 \%$ & $3.99 \%$ \\
Kayacik [9], year 2007 & $90.4 \%$ & $1.38 \%$ \\
Eskin Data Mining [4], year 2002 & $90.00 \%$ & $2.0 \%$ \\
Eskin Clustering [4], year 2002 & $93.00 \%$ & $10.0 \%$ \\
Eskin SVM [4], year 2002 & $98.00 \%$ & $10.0 \%$ \\
Yu [17], year 2008 & $96.02 \%$ & $4.92 \%$ \\
\hline
\end{tabular}

TABLE IV

SUMMARY OF PERFORMANCE COMPARISONS.

$\mathrm{R} 2 \mathrm{~L}$ detection. This is due to the fact that as the system receives more feedback, it becomes less confident in the nodes processing $\mathrm{R} 2 \mathrm{~L}$ connections and thus is able to more accurately identify potential vectors for forwarding.

The integrated approach is exceptional at identifying previously "unknown" attacks. It is intuitive that with the excessive feedback, the accuracy would increase with "unknown" attacks. However, the approach does not require excessive feedback. With the system being corrected on just 282 predictions, it is able to achieve $94.04 \%$ accuracy on "unknown" attacks.

Table IV compares the performance of the integrated AGHSOM approach with eight existing approaches based on machine learning techniques. A-GHSOM is able to consistently produce higher accuracy rates while maintaining low false positive rates. Its false positive rate is lower than all approaches except two approaches proposed in [1] and [9]. Note that the false positive rate is just slightly higher $(1.32 \%$ and $0.42 \%$ ), but the improvement in the major performance metric accuracy is almost $10 \%$ higher. Accuracy and false positive are always tradeoffs. A-GHSOM is able to make the efficient tradeoff, and it is adaptive to a changing problem domain of network intrusion.

\section{Conclusions}

The significance of the A-GHSOM lies in the online adaptation to the ever-changing problem domain of network intrusion and achieving very high accuracy in identifying network intrusions particularly those "unknown" attacks. Experiment results demonstrated the significant impact of each A-GHSOM enhancement on intrusion detection performance. Future work will develop the self-tuning capability of A-GHSOM.

\section{Acknowledgement}

This research was supported in part by U.S. NSF CAREER Award CNS-0844983 and research grant CNS-0720524.

\section{REFERENCES}

[1] Y. Bouzida, F. Cuppens, N. Cuppens-Boulahia, and S. Gombault. Efficient intrusion detection using principal component analysis. In Proc. of the 3eme conference surla securite et architectures reseaux (SAR), 2004

[2] O. Depren, M. Topallar, E. Anarim, and M. K. Ciliz. An intelligent intrusion detection system for anomaly and misuse detection in computer networks. Expert Systems with Applications, 29: 713 - 722, 2005.

[3] M. Dittenbach, D. Merkl, and A. Rauber. The Growing Hiearchical Self Organizing Map. In Proc. of the Int'l Joint Conference on Neural Networks, 2000.

[4] E. Eskin, A. Arnold, M. Prerau, L. Portnoy, and S. Stolfo. A geometric framework for unsupervised anomaly detection: Detecting intrusions in unlabeled data. Applications of Data Mining in Cumputer Security, Chapter 4, 2002.

[5] S. Ganguly, M. Garofalakis, R. Rastogi, and K. Sabnani. Streaming Algorithms for Robust Real-Time Detection of DDoS Attacks. In Proc. of IEEE Int'l Conf. on Distributed Computing Systems (ICDCS), 2007.

[6] K. K. Gupta, B. Nath, and R. Kotagiri. Layered Approach Using Conditional Random Fields for Intrusion Detection. IEEE Trans. on Dependable and Secure Computing, 7(1): 35-49, 2010.

[7] S. Hettich, S. Bay. The UCI KDD archive. University of California, Irvine. http://kdd.ics.uci.edu. 1999.

[8] D. Jiang, Y. Yang, M. Xia. Research on Intrusion Detection Based on an Improved SOM Neural Network. In Proc. of the Fifth Int'l Conference on Information Assurance and Security, 2009.

[9] H. G. Kayacik, Z.-H. Nur, and M. I. Heywood. A hierarchical SOMbased intrusion detection system. Engineering Applications of Artificial Intelligence, Vol. 20, 2007.

[10] Y. Li, B. Fang, L. Guo and Y. Chen. Network anomaly detection based on TCM-KNN algorithm. In Proc. of ACM Symposium on Information, Computer and Communications Security, 2007.

[11] E. Palomo, E. Dominguez, R. Luque, J. Munoz. A new GHSOM Model Applied to Network Security. In Proc. of the 18th Int'l conference on Artificial Neural Networks, 2008.

[12] S. Sarasamma, Q. Zhu, and J. Huff Hierarchical Kohonenen Net for Anomaly Detection in Network Security. IEEE Transaction on systems, man, and cybernetics - part B: Cybernetics, 35(2), 2005

[13] M. G. Schultz, E. Eskin, E. Zadok, and S. J. Stolfo. Data mining methods for detection of new malicious executables. In Proc. of the IEEE symposium on security and privacy, 2001.

[14] S. L. Scott. A Bayesian paradigm for designing intrusion detection systems. Computational Statistics and Data Analysis, 45: 69 - 83, 2004.

[15] G. Shu and D. Lee. Testing security properties of protocol implementations - a machine learning based approach. In Proc. of IEEE Int'l Conf. on Distributed Computing Systems (ICDCS), 2007.

[16] C. Tsai, Y. Hsu, C Lin, and W. Lin. Intrusion detection by machine learning: A review. Expert Systems with Applications, 36(10): 1199412000, 2009.

[17] Z. Yu, J. Tsai, T. Weigert. An adaptive automatically tuning intrusion detection system. In ACM Transactions on Autonomous and Adaptive Systems, 3(3), 2008. 